\title{
Research on the Applied Technology of Testing Grouting Compaction of \\ Sleeves Based on Damped Vibration Method
}

\author{
Li Xiangmin ${ }^{1, a}$, Gao Rundong ${ }^{1, b}$, Wang Zhuolin ${ }^{1, c}$, Zhang Fuwen ${ }^{1, d}$ and Leng Yubing ${ }^{1, e}$ \\ ${ }^{1}$ Shanghai Key Laboratory of Engineering Structure Safety, Shanghai Research Institute of Building \\ Sciences, Shanghai, 200032, China \\ a13601902634@163.com, bgrdong_1@163.com, cwzllzw@163.com, d2004_zhang@tongji.edu.cn, \\ eleng_yb@163.com
}

\begin{abstract}
Keywords: damped vibration method, grouting sleeves, grouting compaction, supplementary grouting
\end{abstract}

Abstract. The damped vibration method is a method for detecting the grouting compaction of sleeves with embedded damping vibration sensors. The damping vibration sensor should be inserted into the grouting outlet as deep as possible before grouting, and testing can begin in 5 10 minutes after finishing grouting. On-site testings indicate: to ensure the compaction degree for the connected cavity grouting, the pressure time should be extended by $10 \sim 15$ seconds before plugging the connected cavity grouting inlet, and the connected cavity grouting inlet should be plugged and fastened at the same time when the grouting pipe is pulled out; when the sleeves are judged to be not compacted through the damped vibration method, supplementary grouting from the initial connected cavity grouting inlet is the first choice.

\section{Introduction}

Monolithic precast concrete structures are being popularized in China. The core issue of such structures is the connection technique of the steel bars. In our country the grouting sleeve connection is used in most of the vertical connection of steel bars in monolithic precast concrete structures. It is found that the sleeves are often grouted poorly. Many reasons may cause this problem, such as the complex construction of the sleeve connection, the concealment of the work, the poor precision during fabrication, the sleeve blockage resulting from improper protective measures, and inadequate training of the operators. The safety of the structure is seriously affected[1-3]. To solve this issue, in addition to the continuous improvement of the construction technology, research on the field inspection of the grouting quality should also be advanced, in order to find out problems in time and take remedial actions. Presently many research institutes are conducting research in this region. Among them, Beijing ZBL Science and Technology Co., Ltd has developed a device for detecting the compaction of the grouting sleeves, which can be used during the grouting process. This paper introduces the application of this detecting method in practical engineering testing, by the cooperation of Shanghai Research Institute of Building Sciences and Beijing ZBL Science and Technology Co., Ltd.

\section{Principle of the Damped Vibration Method}

The damped vibration method is a method for detecting the grouting compaction of sleeves with embedded damping vibration sensors. Vibration of a certain frequency is produced in the sensors under the excitation of specific signals. The amplitude of this vibration reduces with time due to 
friction and medium resistance[4]. In the vibration direction, the forces acting on the object include elastic force and frictional resistance, and the mechanical equation can be expressed as:

$$
x(t)=A_{0} e^{-\beta t} \cos \left(\omega t+\varphi_{0}\right)
$$

In Eq.(1), $x(t)$ is the amplitude at the moment $t ; A_{0}$ is the initial amplitude; $\beta$ is the damping coefficient; $t$ is the time; $\omega$ is the natural angular frequency; and $\varphi_{0}$ is the initial angle.

As can be seen from Eq. (1), the vibration amplitude shows an exponential attenuation. In a constant vibration body, the initial amplitude, natural angular frequency and initial angle are fixed after excitation. The larger the elastic modulus of the medium surrounding the vibrating body is, the larger the damping coefficient is, and the faster the amplitude attenuation should be. As a result, when the medium around the sensor is air and fluid grouting material, the damping coefficient increases successively, so the attenuation of the amplitude increases correspondingly.

According to the above rules, by measuring the amplitude attenuation of the sensors under the excitation signals, the form of the medium around the sensor can be inferred. It can be further determined whether the grouting material has reached the position of the sensors, so as to determine the compaction degree of the grouting sleeves.

During the actual test, the damping vibration sensor is embedded in the bottom of the sleeve outlet before grouting. Tests are conducted before initial-set of the grouting material. By testing the amplitude attenuation of the signals from the sensors, it can be judged whether the sensors are covered by the grouting materials, thus the compaction degree of the grouting sleeves can be determined. In case it is found to be not compact, supplementary grouting should be conducted, so as to achieve quality control during the grouting construction.

\section{On-site testing}

Introduction of the project. A practical project in Shanghai has been selected for on-site testing. It is an monolithic precast shear wall structure. The concrete strength grade is C30, and the strength grade of the longitudinal reinforcement and stirrup is both HRB400. Wall thickness is $200 \mathrm{~mm}$. The sleeves are arranged in a quincunx shape, and connected cavity grouting method is used. The GT18 half-grouting sleeves developed by Beijing Sida Jianmao Technology Development Co., Ltd are adopted, and the CGM-JM-VI grouting material developed also by this company is used. This grouting material features good stability, high strength, good early strength, no shrinkage and good flowability. The mass ratio of water to grouting material is 0.12 . The compressive strength of 28 days is larger than $85 \mathrm{MPa}$. The special plugging material developed by this company is used for plugging the horizontal joints, which is characterized by no shrinkage and cracking resistance, and the compressive strength of 28 days is larger than 50MPa.

The grouting sleeves in two shear walls are tested. One is an interior wall labeled I(the sleeves labeled I-1 I-4). This wall is also used to study the supplementary grouting method at the initial connected cavity grouting inlet. Another is an exterior wall labeled W(the sleeves labeled $\mathrm{W}-1 \sim \mathrm{W}-5)$. The distribution of the sleeves in the walls is shown in Fig. 1, and the on-site testing is shown in Fig. 2. 


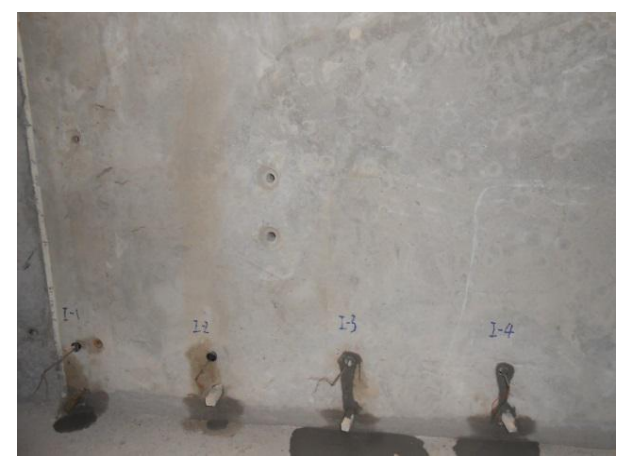

(1) Interior wall I

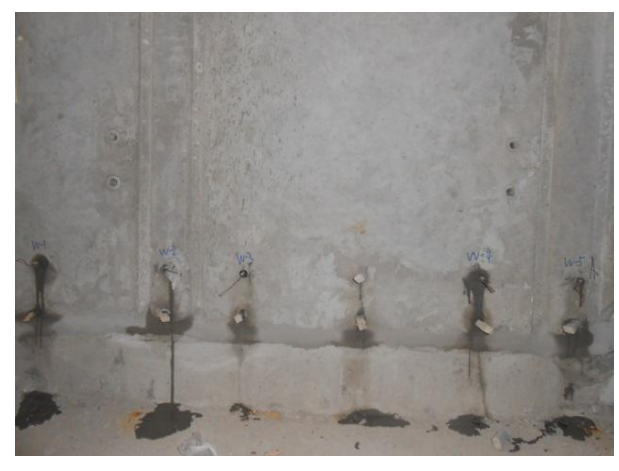

(2) Exterior wall W

Fig. 1 Distribution of the sleeves in the walls

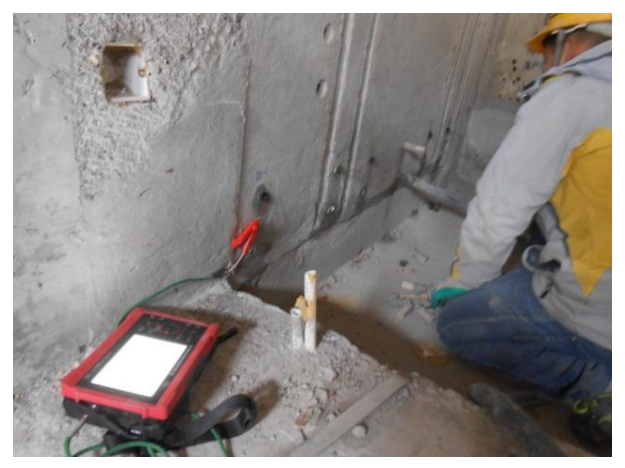

Fig. 2 On-site testing

Testing process. The testing process of the compaction degree of the grouting sleeves in the walls is as follows:

(1) Insert the damping vibration sensor into the sleeve through the outlet as deep as possible. The vent hole of the rubber plug should be on the top center. The center of the tip of the sensor is automatically positioned to be aligned with the center of the grouting outlet.

(2) The walls are grouted through the connected cavity. Generally the bottom grouting inlet of the center sleeve is selected to be the connected cavity grouting inlet. The other bottom grouting inlet are plugged with rubber plugs when the grouting material effuses. When the grouting material outflows from the vent hole of the rubber plug attached to the embedded damping vibration sensor, the vent holes should be plugged with short sticks. At last, when the connected cavity grouting inlet is plugged with rubber plug, the grouting process is finished.

(3) Observe the wave pattern of each sensor after 5 10 minutes, to determine the compaction degree of each sleeve.

\section{Analysis of the test results.}

(1) Interior wall I. The wave patterns from the damping vibration sensors in interior wall I are shown in Fig. 3, and the judging results are listed in Table 1. 


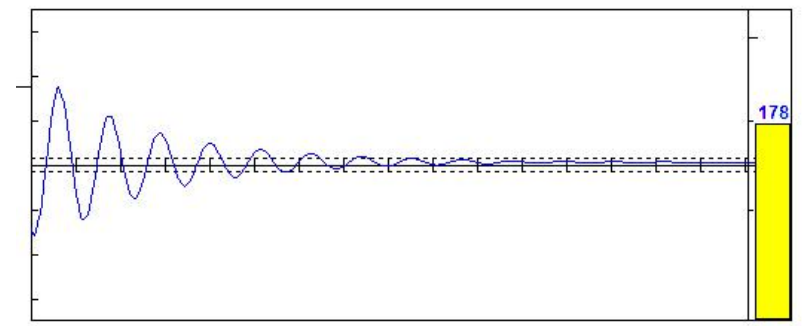

(1) I-1

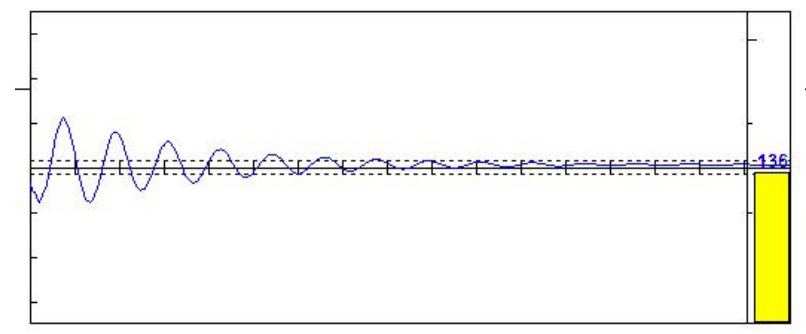

(3) I-3

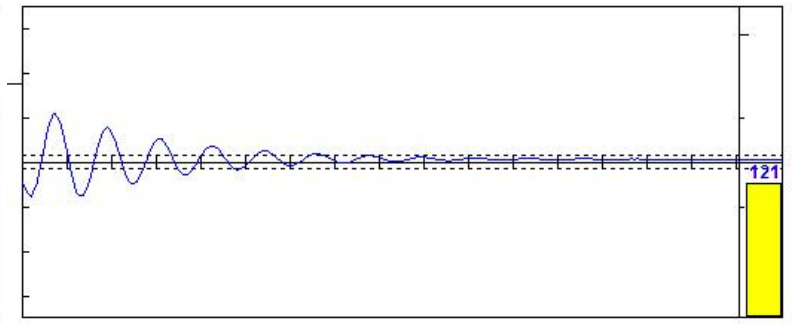

(2) I-2

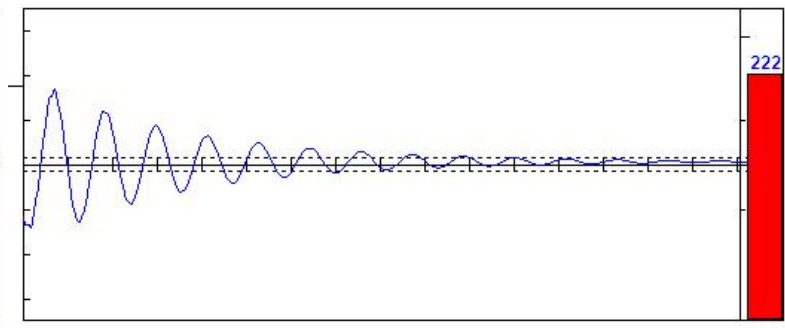

(4) I-4

Fig. 3 Wave patterns from the damping vibration sensors in interior wall I

Table 1 Judging results of each sleeve in interior wall I

\begin{tabular}{|c|c|c|c|c|}
\hline Label of the sleeve & I-1 & I-2 & I-3 & I-4 \\
\hline Energy of vibration & 178 & 121 & 136 & 222 \\
\hline Category & category III & category II & category II & category III \\
\hline Judging results & $\begin{array}{c}\text { Not } \\
\text { compacted }\end{array}$ & $\begin{array}{c}\text { Basically } \\
\text { compacted }\end{array}$ & $\begin{array}{c}\text { Basically } \\
\text { compacted }\end{array}$ & $\begin{array}{c}\text { Not } \\
\text { compacted }\end{array}$ \\
\hline
\end{tabular}

Note: When the energy of vibration $\leq 100$, it is classified as category I; when the energy of vibration $\leq 150$ and $>100$, it is classified as category II; when the energy of vibration $>150$, it is classified as category III. Category I is judged to be compacted, category II is judged to be basically compacted, and category III is judged to be not compacted. Generally, the sleeves of category I and II do not need further management, and the sleeves of category III need supplementary grouting.

According to Fig. 3 and Table 1, the compaction degree of the sleeves in interior wall $\mathrm{I}$ is unsatisfactory. It is mainly due to insufficient pressure time before plugging the connected cavity grouting inlet and plugging the grouting inlet not timely.

Supplementary grouting is conducted from the initial connected cavity grouting inlet, and tests are conducted after 10 minutes. The wave patterns from the damping vibration sensors in interior wall I after supplementary grouting are shown in Fig. 4, and the judging results are listed in Table 2. 


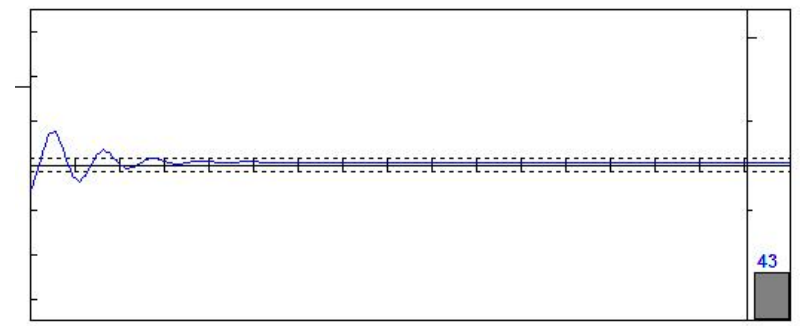

(1) I-1

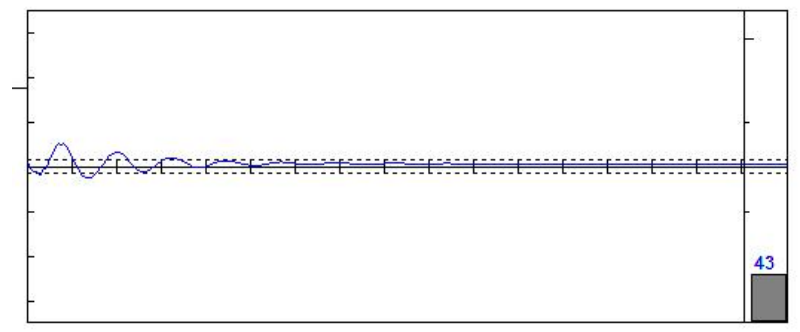

(3) I-3

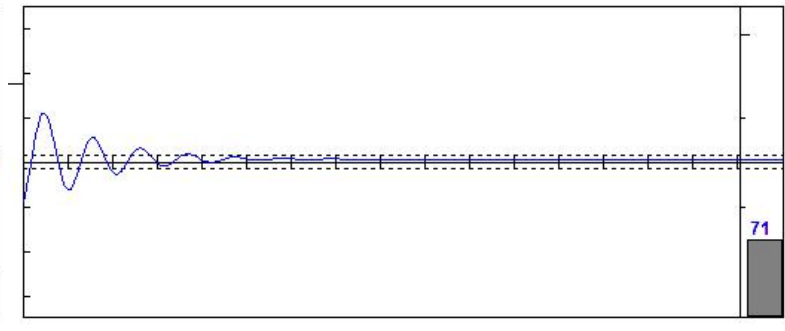

(2) I-2

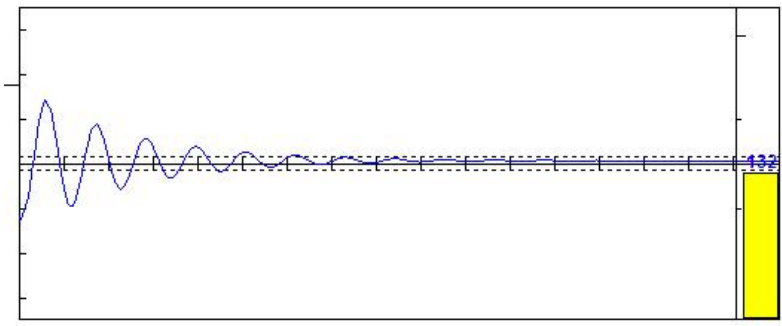

(4) I-4

Fig. 4 Wave patterns from the damping vibration sensors in interior wall I after supplementary grouting

Table 2 Judging results of each sleeve in interior wall I after supplementary grouting

\begin{tabular}{|c|c|c|c|c|}
\hline Label of the sleeve & I-1 & I-2 & I-3 & I-4 \\
\hline Energy of vibration & 43 & 71 & 43 & 132 \\
\hline Category & category I & category I & category I & category II \\
\hline Judging results & Compacted & Compacted & Compacted & $\begin{array}{c}\text { Basically } \\
\text { compacted }\end{array}$ \\
\hline
\end{tabular}

According to Fig. 4 and Table 2, after supplementary grouting through the initial connected cavity grouting inlet, the compaction degree of the sleeves in interior wall I can meet the requirement.

(2) Exterior wall W. The wave patterns from the damping vibration sensors in exterior wall W are shown in Fig. 5, and the judging results are listed in Table 3. 


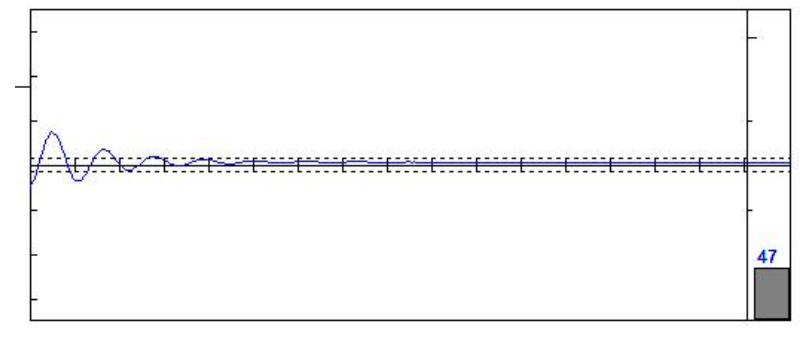

(1) W-1

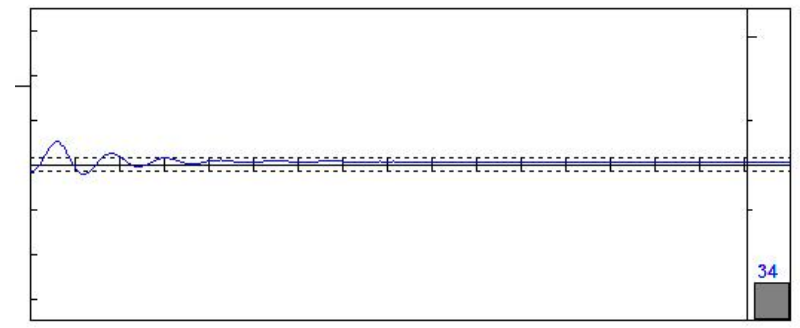

(3) W-3

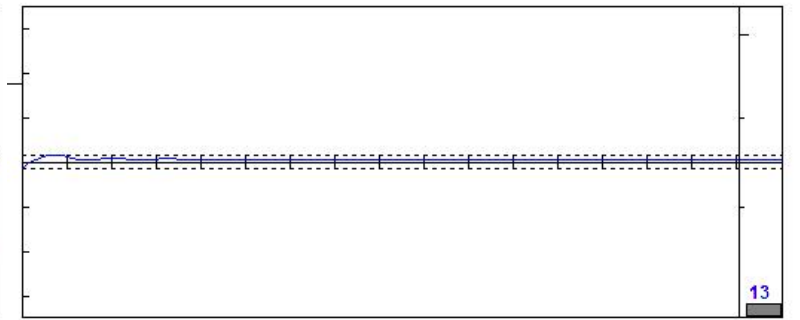

(2) W-2

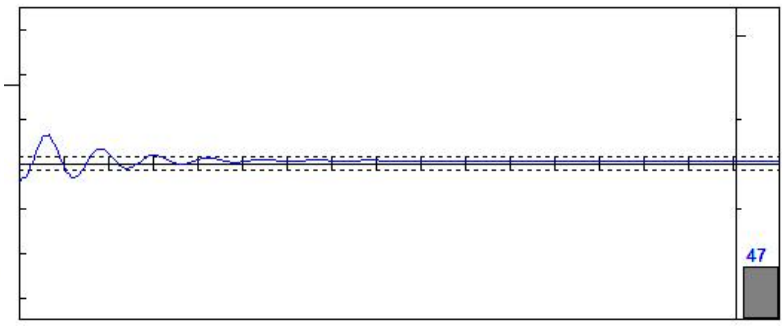

(4) W-4

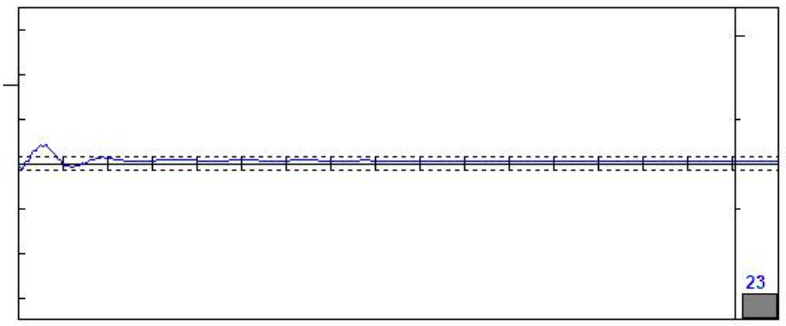

(5) W-5

Fig. 5 Wave patterns from the damping vibration sensors in exterior wall $W$

Table 3 Judging results of each sleeve in exterior wall $W$

\begin{tabular}{|c|c|c|c|c|c|}
\hline Label of the sleeve & W-1 & W-2 & W-3 & W-4 & W-5 \\
\hline Energy of vibration & 47 & 13 & 34 & 47 & 23 \\
\hline Category & category I & category I & category I & category I & category I \\
\hline Judging results & Compacted & Compacted & Compacted & Compacted & Compacted \\
\hline
\end{tabular}

According to Fig. 5 and Table 3, all the sleeves in wall $\mathrm{W}$ are compacted. Based on the grouting experience of the interior wall $\mathrm{I}$, during the grouting process of exterior wall $\mathrm{W}$, the grouting outlets and inlets are plugged and fastened in time after leakage. Before plugging the connected cavity grouting inlet, the pressure time is extended by 10 15 seconds. Finally, when the grouting pipe is pulled out, the connected cavity grouting inlet is plugged and fastened at the same time.

\section{Conclusions}

(1) The damped vibration method is a method for detecting the grouting compaction of sleeves with embedded damping vibration sensors. During the test, the center of the tip of the sensor should be kept to be aligned with the center of the grouting outlet. The damping vibration sensor should be inserted into the grouting outlet as deep as possible, and the rubber plug at the end of the sensor should be fastened. Generally test can begin in 5 10 minutes after finishing grouting, when the grouting material has reached each small gap.

(2) To ensure the compaction degree, during the connected cavity grouting process, when the 
grouting material outflows from the grouting outlets or grouting inlets, the outlets or inlets should be plugged and fastened timely. Before plugging the connected cavity grouting inlet, the pressure time should be extended by 10 15 seconds. Finally, the operators processing the grouting and plugging operations should cooperate closely. The connected cavity grouting inlet should be plugged and fastened at the same time when the grouting pipe is pulled out.

(3) When the sleeves are judged to be not compacted through the damped vibration method, for the sleeves grouted with connected cavity, supplementary grouting from the initial connected cavity grouting inlet is the first choice. If infeasible, supplementary grouting can be conducted through the grouting inlets that are not compacted.

\section{Acknowledgements}

Support for this work is provided by the project from Science and Technology Commission of Shanghai Municipality (No. 15DZ1203506).

\section{References}

[1] Qi Baoku, Wang Dan, Bai Shu, et al. Common Quality Problems and Preventive Measures of Prefabricated Construction[J]. Construction Economy, 2016, Vol. 37(5): 28-30.

[2] Su Yangyue, Zhao Jinkai, Xu Youquan, et al. Research on Quality Problems and Improvement of Production and Construction of Prefabricated Building[J]. Construction Economy, 2016, Vol. 37(11): 43-48.

[3] Chang Chunguang, Wang Jiayuan, Li Hongxue. Identification and Control of Quality Elements for Prefabricated Concrete Constructions[J]. Journal of Shenyang Jianzhu University (Social Science), 2016, Vol. 18(1): 58-63.

[4] Dai Depei. Engineering Application of Damping Technology[M]. Beijing: Tsinghua University Press, 1991. 